\title{
A Wiener Filtering Approach over the Euclidean Motion Group for Radon Transform Inversion
}

\author{
Can Evren Yarman and Birsen Yazıcı \\ Drexel University, 3141 Chestnut Street, Philadelphia, PA
}

\begin{abstract}
The problem of Radon transform inversion arises in fields as diverse as medical imaging, synthetic aperture radar, and radio astronomy. In this paper, we model the Radon transform as a convolution integral over the Euclidean motion group and provide a novel deconvolution method for its inversion. The deconvolution method presented here is a special case of the Wiener filtering framework in abstract harmonic analysis that was recently developed by the author. The proposed deconvolution method provides a fundamentally new statistical formulation for the inversion of the Radon transform that can operate in nonstationary noise and signal fields. It can be utilized for radiation treatment planning, inverse source problems, and 3D and 4D computed tomography. Furthermore, it is directly applicable to many computer vision and pattern recognition problems, as well as to problems in robotics and polymer science. Here, we present an algorithm for the discrete implementation of the Wiener filter and provide a comparison of the proposed image reconstruction method with the filtered back projection algorithms.
\end{abstract}

Keywords: Wiener filtering, noncommutative harmonic analysis, Euclidean motion group

\section{INTRODUCTION}

In X-ray computed tomography, an X-ray beam with known energy is sent trough an object and attenuated $\mathrm{X}$-rays are collected by an array of detectors. The attenuation in the final X-ray beam provides a means of determining the mass density of the object along the path of the X-ray. In $2 \mathrm{D}$, the relationship between the attenuation and mass density along the X-ray path at angle $\theta$ and radius $t$ is given by the Radon transform:

$$
\mathcal{R} f\left(R_{\theta}, t\right)=P(\theta, t)=\int_{\mathbb{R}^{2}} f\left(x_{1}, x_{2}\right) \delta\left(t-\cos \theta x_{1}-\sin \theta x_{2}\right) d x_{1} d x_{2},
$$

where $\delta$ is the Dirac delta function, $f$ is the unknown attenuation coeffients, and $R_{\theta}$ is the rotation matrix in counter clockwise direction. Similarly, in positron emission tomography, single photon emission computed tomography and synthetic aperture radar, the line projections and the unknown image are related by the Radon transform.

In the last two decades, Radon transform inversion has received a great deal of attention. For recent works, $\mathrm{see}^{2,3,5,7,11}$. Here, we formulate the Radon transform as a convolution integral over the Euclidean motion group and propose a deconvolution method based on a novel Wiener filtering approach in harmonic analysis that was recently developed by the author ${ }^{16}$. The method provides a minimum mean square error (MMSE) regularized solution for the deconvolution problem. It has a number of advantages as compared to standard Radon transform inversion methods. It is fundamentally a new statistical formulation of the Radon transform inversion that can operate in nonstationary noise and signal fields. It can be easily extended to Radon transform inversion in higher dimensions. The formulation allows application of the method to radiation therapy treatment planning, and non-rigid body motion imaging in X-ray computed tomography. Furthermore, it is applicable to other deconvolution problem over the Euclidean motion group, in particular, to object recognition and tracking in computer vision, as well as to problems in robotics and polymer science ${ }^{4,6}$.

Mathematically, Euclidean motion group, $S E(2)$, in two dimension is the group formed by the semidirect product of the additive group of $\mathbb{R}^{2}$ and the rotation group $S O(2)$. Each element consists of a translation and

Further author information: C.E.Y.: E-mail: cey22@drexel.edu, B.Y.: E-mail: byazici@cbis.ece.drexel.edu 
a rotation component. The group operation can be viewed as a rotation with respect to an origin followed by a translation. Group convolution can be viewed as a generalization of the ordinary convolution in which the additive group translation is replaced by a group translation, i.e., $f(-y+x) \rightarrow f\left(g^{-1} h\right)$. An important property of the group convolution is that it is mapped to a multiplication in the group Fourier transform domain. Group Fourier transforms along with stochastic processes that are invariant with respect to group operation form the key components of the proposed deconvolution method.

The paper is organized as follows: In Section 2, we provide some preliminaries for the Euclidean motion group, its unitary representations and the associated Fourier transform. In Section 3, we present the convolution integral representation of the Radon transform over $S E(2)$. In Section 4, we introduce the Wiener filtering framework for the solution of the convolution integral over $S E(2)$. In Section 5, we present an algorithm for the discrete implementation of the Wiener filtering. In Section 6, we present simulation experiments to demonstrate the preformance of the proposed method with respect to several construction parameters and filtered back projection (FBP). Section 7 includes concluding remarks and future work.

\section{PRELIMINARIES}

\subsection{Euclidean Motion Group}

Euclidean motion group is a locally compact topological group. Each of its elements consists of a translation and a rotation parameter. A formal definition of the motion group is given as follows.

DEFINITION 2.1. The subgroup $G$ of real valued invertible matrices, consisting of the elements

$$
g(\vec{r}, \theta)=\left(\begin{array}{ccc}
\cos \theta & -\sin \theta & \vec{r} \\
\sin \theta & \cos \theta & 1 \\
& 0 & 1
\end{array}\right)
$$

for any $\theta \in[0,2 \pi)$ and for any $\vec{r}=\left(r_{1}, r_{2}\right)^{T} \in \mathbb{R}^{2}$ is called the motion group of the Euclidean plane, and denoted by $S E(2)$. Operation of the group is the usual matrix multiplication.

Note that $\vec{r}$ denotes a vector, and $r$ denotes a scalar.

$S E(2)$ group action can be viewed as a rotation operation followed by a translation operation on the plane, i.e., $g$ translates $\left(x_{1}, x_{2}\right)^{T}$ to $\left(x_{1}^{\prime}, x_{2}^{\prime}\right)^{T}$ in the following fashion:

$$
\left.\begin{array}{l}
x_{1}^{\prime}=x_{1} \cos \theta-x_{2} \sin \theta+r_{1} \\
x_{2}^{\prime}=x_{1} \sin \theta+x_{2} \sin \theta+r_{2}
\end{array}\right\} \rightarrow\left(x_{1}^{\prime}, x_{2}^{\prime}\right)=g \cdot\left(x_{1}, x_{2}\right) .
$$

Let $L^{2}(S E(2))$ denote the Hilbert space of the square integrable complex valued functions. Let $f_{1,2}$ : $S E(2) \rightarrow \mathbb{C}$, be two functions in this space. Then, the inner product of $f_{1}$ and $f_{2}$ is given by

$$
\left(f_{1}, f_{2}\right)=\int_{S E(2)} \overline{f_{1}(g)} f_{2}(g) d \mu(g)
$$

where $d \mu(g)$ is the invariant Haar measure on $S E(2)$, and is given by

$$
d \mu(g)=\frac{1}{(2 \pi)^{2}} r d r d \phi d \theta
$$

and $(r, \phi)$ is the polar coordinate representation for $\left(r_{1}, r_{2}\right)$. Note that Equation 3 can be used to define the action of $S E(2)$ over the square integrable functions of $\mathbb{R}^{2}$ and $S E(2)$ as follows:

$$
\begin{aligned}
L_{q}(g) f(x) & =f\left(g^{-1} \cdot x\right) \\
L(g) f(h) & =f\left(g^{-1} h\right)
\end{aligned} .
$$

Here $L_{q}$ and $L$ are called the quasi left and left regular representations of $S E(2)$, respectively. The convolution integral is defined in terms of left regular representation of the group as follows:

$$
\left(f_{1} *_{S E(2)} f_{2}\right)(g)=\int_{S E(2)} f_{1}(h) f_{2}\left(h^{-1} g\right) d \mu(h) .
$$




\subsection{Unitary representations of $\operatorname{SE}(2)$}

The unitary representation $U^{p}, p$ a nonnegative real number, of $S E(2)$ of the square integrable functions on the unit cirle, i.e., $L^{2}(\mathbb{R} / 2 \pi \mathbb{Z})$, is given by

$$
\left(U_{g}^{p} F\right)(s)=e^{-i p(\vec{r} \cdot \vec{s}))} F\left(R(\theta)^{-1} s\right), \quad p \in \mathbb{R}^{+} \cup\{0\}
$$

where $g=(\vec{r}, \theta) \in S E(2)$ and $(\vec{r} \cdot \vec{s})$ is the usual inner product in $\mathbb{R}^{2}$.

Any function $f$ defined on the unit circle can be written as a weighted sum of orthonormal basis functions as $f(\psi)=\sum_{n \in \mathbb{Z}} a_{n} e^{i n \psi}$. Thefore, the matrix elements of the unitary representations can be expressed as follows:

$$
u_{m n}(g, p)=\left(e^{i m \psi}, U_{g}^{p} e^{i n \psi}\right)=\frac{1}{2 \pi} \int_{0}^{2 \pi} e^{-i m \psi} e^{-i\left(r_{1} p \cos \psi+r_{2} p \sin \psi\right)} e^{i n(\psi-\theta)} d \psi, \quad \forall m, n \in \mathbb{Z} .
$$

Note that the matrix representation of the unitary representations $U_{g}^{p}$ are infinite dimensional. Another way of expressing the matrix elements of representations is by means of Bessel functions:

$$
u_{m n}(g(r, \phi, \theta), p)=i^{n-m} e^{-i[n \theta+(m-n) \phi]} J_{n-m}(p r)
$$

where $J_{v}(z)$ is the $v^{\text {th }}$ order Bessel function. Using the expression in Equation 10, one can show that

$$
\begin{aligned}
u_{m n}\left(g^{-1}, p\right) & =u_{m n}^{-1}(g, p)=\overline{u_{n m}(g, p)} \\
& =i^{n-m} e^{i[m \theta+(n-m) \phi]} J_{m-n}(p r) .
\end{aligned}
$$

It is straightforward to show that the matrix elements satisfy the following symmetry relationship:

$$
\overline{u_{m n}(g, p)}=(-1)^{m-n} u_{-m,-n}(g, p) .
$$

\subsection{Fourier Transform over $\mathrm{SE}(2)$}

DEFINITION 2.2. Let $U^{p}$ be a unitary representation of $S E(2)$. For any complex valued function $f \in L^{2}(S E(2))$ the Fourier transform over $S E(2)$ is defined as

$$
\mathcal{F}(f)=\hat{f}(p)=\int_{S E(2)} f(g) U_{g^{-1}}^{p} d \mu(g),
$$

where $d \mu(g)=\frac{1}{(2 \pi)^{2}} r d r d \phi d \theta, 0 \leq p \in \mathbb{R}$, and $\int_{S E(2)}=\int_{0}^{2 \pi} \int_{\mathbb{R}^{2}}$. The corresponding inverse Fourier transform is given by

$$
\left(F^{-1}\right)(\hat{f})=f(g)=\int_{0}^{\infty} \operatorname{trace}\left(\hat{f}(p) U_{g}^{p}\right) p d p .
$$

The matrix elements of the Fourier transform $\hat{f}(p)$ can be expressed as follows:

$$
\hat{f}_{m n}(p)=\left(e^{i m \psi}, \hat{f}(p) e^{i n \psi}\right)=\int_{S E(2)} f(g) u_{m n}\left(g^{-1}, p\right) d \mu(g) .
$$

Similarly, the inverse Fourier transform can be expressed in terms of the matrix elements of $f, \hat{f}$ and $U^{p}$

$$
f(g)=\sum_{n, m \in Z} \int_{0}^{\infty} \hat{f}_{m n}(p) u_{n m}(g, p) p d p .
$$

The inverse Fourier transform shows the decomposition of $f(g)$ in terms of its projections into G-invariant subspaces. Similar to the Fourier transform over $\mathbb{R}^{n}$, an analogue of the Parsevals equality for Fourier transform over $S E(2)$ exists.

THEOREM 2.3. Let $f, f_{1}, f_{2}$ be square integrable functions on $G$, then the following properties hold. 
1.

$$
\int_{G}|f(g)|^{2} d \mu(g)=\int_{0}^{\infty}\|\hat{f}(p)\|_{2}^{2} p d p
$$

This equality is known as the Plancherel equality.

2.

$$
\mathcal{F}\left(f_{1} * f_{2}\right)=\mathcal{F}\left(f_{2}\right) \mathcal{F}\left(f_{1}\right)
$$

This is the convolution property of the Fourier transform.

3.

$$
\left(f^{*}\right)^{\wedge}(p)=(\hat{f}(p))^{\dagger}, \text { where } f^{*}(g)=\overline{f\left(g^{-1}\right)} .
$$

where ${ }^{\dagger}$ denotes the adjoint operation. In matrix form adjoint operation corresponds to complex conjugate operation.

For a detailed discussion of group representation theory and the group Fourier transforms, the reader is referred to ${ }^{13,14}$.

\section{RADON TRANSFORM AS A CONVOLUTION INTEGRAL OVER SE(2)}

In this section, we provide a formulation of the Radon transform equation as a convolution integral over $\mathrm{SE}(2)$. This formulation is based on the extension of functions from $\mathbb{R}^{2}$ and $\mathbb{R} \times S O(2)$ to $\mathrm{SE}(2)$. Let $g, h \in S E(2)$ with $g=g(\vec{r}, \theta), h=h(\vec{x}, \phi)$, where $\vec{r}=\left(r_{1}, r_{2}\right)^{T}$ and $\vec{x}=\left(x_{1}, x_{2}\right)^{T}$.

$$
\begin{aligned}
f_{1} * f_{2}^{*}(g) & =\int_{S E(2)} f_{1}(h) f_{2}\left(g^{-1} h\right) d \mu(h) \\
& =\int_{S E(2)} f_{1}(g h) f_{2}(h) d \mu(h) \\
=f_{1} * f_{2}^{*}(\vec{r}, \theta) & =\int_{S E(2)} f_{1}(R(\theta) \vec{x}+\vec{r}, \theta+\phi) f_{2}(\vec{x}, \phi) d \mu(h) .
\end{aligned}
$$

Choosing $f_{1}(\vec{x}, \phi)=\delta\left(e_{1} \cdot \vec{x}\right), f_{2}(\vec{x}, \phi)=f(-\vec{x})$ the convolution in Equation 22 results in

$$
f_{1} * f_{2}^{*}(g)=\mathcal{R} f\left(-\theta,-r_{1}\right)=f_{3}(g)
$$

where $e_{1}=(1,0)^{T}$ is the unit vector in $x$ direction and $\cdot$ is the usual inner product. Therefore $e_{1} \cdot \vec{x}=x_{1}$. Equivalently, Equation 23 can be rewritten as

$$
f_{2} * f_{1}^{*}(g)=f_{3}^{*}
$$

Thus, Radon transform inversion can be viewed as a deconvolution problem over $\mathrm{SE}(2)$.

\section{STATIONARY PROCESS AND WIENER FILTERING OVER GROUPS}

Let $x(t), t \in T$ be a stochastic process. The covariance function of $x(t), t \in T, \gamma: G \times G \rightarrow \mathbb{C}$ is given by

$$
\gamma(s, t)=E[x(s), x(t)] .
$$

Let group $\mathrm{G}$ act transitively on $T$ and let $x(t), t \in T$ satisfy the following condition:

$$
\gamma(g s, g t)=\gamma(s, t), g \in G \text {. }
$$

Then $x(t), t \in T$ is called a wide sense stationary stochastic process. ${ }^{9}$ Choosing $T$ as the group $G$ itself, the action of the group becomes the group operation. For the rest of this paper, we assume that $T=G$. In this case, the covariance function $\gamma$ of the group satisfies

$$
\gamma(g, h)=\gamma\left(h^{-1} g, e\right), g, h \in G .
$$


where $e$ is the identity element of the group $G$. Substituting $g h^{-1}$ for $h$,

$$
\gamma\left(g, g h^{-1}\right)=\gamma(h, e), g, h \in G .
$$

Thus, the covariance function of a wide sense stationary process reduces to a function on $G$. For notational convenience we denote $\gamma(g, e)$ by $\gamma(g)$. In the case of Euclidean motion group, the wide sense stationarity implies a translational and rotational invariance.

Note that $\gamma(g)$ is a nonnegative function over $G$. Similar to the classical spectral density function, one can define a spectral density function for $x(g), g \in G$ via group Fourier transform. Under some conditions on $\gamma$, we define $^{16}$

$$
S(p)=\int_{G} \gamma(g) U_{g}^{p} d \mu(g)
$$

as the spectral density function of $x(g), g \in G$. Note that $S(p)$ is a non-negative definite, Hermitian operator.

THEOREM 4.1. Let $x(g), n(g)$ be two zero mean, wide sense stationary processes over $S E(2)$. Let the observation $y(g)$ be given as

$$
y(g)=x *_{S E(2)} f(g)+n(g)=\int_{S E(2)} x(h) f\left(h^{-1} g\right) d \mu(h)+n(g)
$$

and

$$
E[x(g) n(g))]=0
$$

where $f \in L^{2}(S E(2))$. Then the linear filter, $W_{\text {opt }}$, minimizing the error function

$$
J=\int_{G} E\left[|x(g)-\tilde{x}(g)|^{2}\right] d \mu(g)
$$

is group invariant, i.e.,

$$
\tilde{x}=\int_{S E(2)} y(h) W_{o p t}\left(h^{-1} g\right) d \mu(h)
$$

and the Fourier transform, $\hat{W}_{\text {opt }}$, of the optimal filter is given by

$$
\hat{W}_{\text {opt }}(p)=S_{x}(p) \hat{f}^{\dagger}(p)\left[\hat{f}(p) S_{x}(p) \hat{f}^{\dagger}(p)+S_{n}(p)\right]^{-1}
$$

The spectral density function of the associated mean square error is given by

$$
S_{e}(p)=\left(I-\hat{W}_{o p t}(p) \hat{f}(p)\right) S_{x}(p) .
$$

Proof. The proof is provided in ${ }^{16}$.

Note that in the absence of noise and prior information about the signal, the filter takes the following form

$$
\hat{W}_{o p t}(p)=\hat{f}^{\dagger}(p)\left[\hat{f}(p) \hat{f}^{\dagger}(p)\right]^{-1},
$$

which is nothing but the pseudo inverse of $\hat{f}$. Since $\hat{f}$ is a trace class operator, its inverse is unbounded. The inversion can be stabilized by a zero order Tikhonov regularization which amounts to the additive white noise assumption in the Wiener filtering framework.

$$
\hat{W}_{o p t}(p)=\hat{f}^{\dagger}(p)\left[\hat{f}(p) \hat{f}^{\dagger}(p)+\sigma^{2} I(p)\right]^{-1} .
$$

\section{AN ALGORITHM FOR THE DISCRETE IMPLEMENTATION OF THE WIENER FILTERING OVER SE(2)}

In this section, we shall describe an algorithm for the discrete implementation of the Wiener filtering over $S E(2)$ and apply this algorithm to the inversion of Radon transform. Discrete implementation requires discritization of the motion group, approximate implementation of the Fourier transform and band-limited approximation of functions in $L^{2}(S E(2))$. 


\subsection{Discrete Motion Subgroup}

The subgroup of $S E(2)$ consisting of elements $g=\left(\vec{r}, \theta_{i}\right)$ where $\theta_{j}=\frac{2 \pi j}{N}, N \in \mathbb{Z}^{+}, j=0 \ldots N-1$, is called the discrete motion group of the plane. This group is the semidirect product of the group of rotational symmetries of a regular $N$-gon, denoted by $C^{N}$, and the additive group of $\mathbb{R}^{2}$. When the integral over $S O(2)$ is discretized at $N$ equally spaced intervals, the integration is approximated by a sum over the discrete group $C^{N}$ as follows:

$$
\int_{S O(2)} f(\cdot, \theta) d \mu(\theta)=\frac{1}{2 \pi} \int_{0}^{2 \pi} f(\cdot, \theta) d \theta \rightarrow \frac{1}{N} \sum_{j=0}^{N-1} f\left(\cdot, \theta_{j}\right) \quad \theta_{j}=\frac{2 \pi j}{N} .
$$

Choosing a pulse orthonormal basis on the unit circle,

$$
b_{n}(\theta)=\left\{\begin{array}{cc}
N^{1 / 2} & \theta \in\left[\frac{2 \pi n}{N}, \frac{2 \pi(n+1)}{N}\right) \\
0 & \text { otherwise }
\end{array}, n=0 \ldots N-1,\right.
$$

the matrix elements of the irreducible unitary representations are given by ${ }^{12}$

$$
u_{m n}(g, p)=\left(b_{m}(\psi), U_{g}^{p} b_{n}(\psi)\right)=\frac{1}{2 \pi} \int_{0}^{2 \pi} b_{m}(\psi) e^{-i\left(r_{1} p \cos \psi+r_{2} p \sin \psi\right)} b_{n}(\psi-\theta) d \psi
$$

where $g=(\vec{r}, \theta)$. Due to the orthogonality of basis functions, Equation 40 can be reexpressed as

$$
\left.u_{m n}\left(g_{j}\right), p\right)=e^{i p\left(\vec{r} \cdot \overrightarrow{u_{m}}\right)} \delta_{m-j, n},
$$

where $g_{j}=g\left(\vec{r}, \theta_{j}\right), \vec{u}_{m}=\left(\cos \left(\frac{2 \pi m}{N}\right), \sin \left(\frac{2 \pi m}{N}\right)\right)^{T}$, and $\delta_{m, n}$ is the Kronecker delta function.

\subsection{Approximate Fourier Transform over SE(2)}

A fast algorithm for the Fourier Transform over $S E(2)$ based on fast Fourier tranforms (FFT) was given in ${ }^{12}$. This algorithm is based on step wise computation of the integral of $\hat{f}_{m n}$ using the inner product expression for matrix elements of unitary representations, given in Equation 9,

$$
\hat{F}_{m n}(p)=\int_{r \in \mathbb{R}^{2}} \int_{\theta=0}^{2 \pi} \int_{\psi=0}^{2 \pi} F(\vec{r}, \theta) e^{i n \psi} e^{i(\vec{p} \cdot \vec{r})} e^{-m(\psi-\theta)} d^{2} \vec{r} d \theta d \psi
$$

where $\vec{p}=(p \cos \psi, p \sin \psi)$. For numerical implementation, the data has to be discritized. Let $S$ be the number of harmonics for the computation of $S E(2)$ Fourier transform, i.e., $m, n=-S, \ldots, S$. Then, we can choose the number of discrete samples of $F$ on $S E(2)$ as shown in Table 1.

\begin{tabular}{lll}
$N_{R}$ & Number of samples on $S O(2)$ & $O(S)$ \\
$N_{\vec{r}}$ & Number of samples on $\mathbb{R}^{2}$ & $O\left(S^{2}\right)$ \\
$N$ & Total number of samples on $S E(2)=N_{\vec{r}} N_{R}$ & $O\left(S^{3}\right)$ \\
\hline$N_{p}$ & Number of samples on $p$ interval & $O(S)$ \\
$N_{\psi}$ & Number of samples on $[0,2 \pi)$ & $O(S)$ \\
$N_{m n}$ & Total number of harmonics $=N_{R} N_{\psi}$ & $O\left(S^{2}\right)$
\end{tabular}

Table 1. Sampling in $S E(2)$ as an order of $S, O(S)$.

The integral in Equation 42 may be computed in 4 steps:

1.

$$
F_{1}(\vec{p}, \theta)=\int_{\mathbb{R}^{2}} F(\vec{r}, \theta) e^{i(\vec{p} \cdot \vec{r})} d^{2} \vec{r},
$$

2D inverse Fourier transform of $F$ with respect to $\vec{r}$, using FFT takes $O\left(N_{R} N_{\vec{r}} \log \left(N_{\vec{r}}\right)\right)=O\left(N \log \left(N_{\vec{r}}\right)\right)$ number of computations. 
2. Interpolation of $F_{1}(p, \psi, \theta)=F_{1}(\vec{p}, \theta)$ from cartesian coordinates to polar coordinates for each $\theta$,

$$
F_{1}(p, \psi, \theta)=\text { interpolate }\left\{F_{1}(\vec{p}, \theta)\right\},
$$

takes $O\left(N_{R} N_{\vec{r}} \log \left(N_{\psi} N_{p}\right)\right)=O\left(N \log \left(N_{\psi} N_{p}\right)\right)$ number of computations. ${ }^{1}$

3.

$$
F_{2}^{(m)}(p, \psi)=\int_{S O(2)} F_{1}(p, \psi, \theta) e^{i m \theta} d \theta
$$

inverse Fourier transform along $\theta$, takes $O\left(N_{p} N_{\psi} N_{R} \log \left(N_{R}\right)\right)=O\left(N_{p} N_{m n} \log \left(N_{R}\right)\right)$ number of computations.

4.

$$
\hat{F}_{m n}(p)=\int_{o}^{2 \pi}\left[F_{2}^{(m)}(p, \psi) e^{-i m \psi}\right] e^{i n \psi} d \psi
$$

inverse Fourier transform along $\psi$, takes $O\left(N_{R} N_{p} N_{\psi} \log \left(N_{\psi}\right)\right)=O\left(N_{p} N_{m n} \log \left(N_{\psi}\right)\right)$ number of computations.

Let the number of sampes on $S O(2)$ be in the order of $S$, then the computational complexity of $S E(2)$ Fourier transform is $O\left(S^{3} \log \left(S^{3}\right)\right)$.

\subsection{The concept of bandlimitedness for functions over $\operatorname{SE}(2)$}

Note that the Fourier transform of a function over $S E(2)$ is a family of linear operators that are not necessarily finite dimensional. For numerical implementation and processing in Fourier domain, it is necessary to approximate these linear operators as finite matrices. Furthermore, $S E(2)$ has infinitely many irreducible representations, i.e, $U^{p}$, a nonnegative $p \in \mathbb{R}$. We assume that a function over $S E(2)$ is band limited if it satisfies the following conditions:

- The spectral components $\hat{f}(p)$ is non zero only for some $p<B$.

- If the eigenvalues of the non-zero spectral components decay to zero at a rate that is fast enough that all spectral components can be approximated by finite matrices.

These assumptions are inherent in the FFT based implementation of the Wiener filtering and minimum mean square error image reconstruction.

\section{4. $\mathrm{SE}(2)$ Fourier Transform of a function on $\mathbb{R}^{2}$}

If $f(g)$ is independent of $\theta$, i.e., $f(g)=f\left(r_{1}, r_{2}\right)$, one can show that the matrix elements of $\hat{f}$ is independent of the rows, i.e., $\hat{f}_{m n}=\hat{f}_{q n}$. This propery can be observed from the inverse Fourier transform of the discrete Euclidean motion group.

$$
\hat{f}_{m n}=\sum_{j=0}^{N-1} \int_{\mathbb{R}^{2}} f\left(\vec{r}, \theta_{j}\right) e^{-i p\left(\vec{r} \cdot \overrightarrow{u_{n}}\right)} \delta_{n-j, m} d^{2} \vec{r} .
$$

Therefore, it is sufficient to compute one row of each spectral component. Recall that, in Equation 24 both $f_{1}$ and $f_{2}$ are $\theta$ invariant functions. Let $F_{1}, F_{2}$ and $F_{3}$ be the matrix representations for the Fourier transforms of $f_{1}, f_{2}$ and $f_{3}$ for a fixed $p=p_{0}$, respectively. Let $\hat{f}_{m n}$ denote $\hat{f}_{m n}\left(p_{0}\right)$, and $\left[\hat{f}_{m n}\right]$ a column vector, with elements equal to $\hat{f}_{m n}$, i.e., $\left[\hat{f}_{m n}\right]=\left[\ldots \hat{f}_{m n} \hat{f}_{m n} \ldots\right]^{T}$. Then, for $p=p_{0}$, Fourier domain equivalent of Equation 24 can be rewritten as follows:

$$
F_{1}^{\dagger} F_{2}=F_{3}^{\dagger}
$$


where

$$
F_{1}=\left[\begin{array}{lllll}
\cdots & {\left[\hat{f}_{10,-1}\right]} & {\left[\hat{f}_{10,0}\right]} & {\left[\hat{f}_{10,1}\right]} & \cdots
\end{array}\right],
$$

is a columnwise representations for $F_{1}$. As mentioned earlier, in the bandlimited case the matrices are approximated with a finite number of harmonics, i.e., $m, n=-S, \ldots, S$. Expanding Equation 49, the bandlimited approximation of Equation 48 can be expressed as

$$
\left[\begin{array}{ccc}
\overline{\hat{f}_{10,-S}} & & 0 \\
& \ddots & \\
0 & & \overline{\hat{f}_{10, S}}
\end{array}\right]\left[\begin{array}{ccc}
1 & \cdots & 1 \\
\vdots & \ddots & \vdots \\
1 & \cdots & 1
\end{array}\right] \tilde{F}_{2}=\left[\begin{array}{ccc}
\overline{\hat{f}_{3-S, S}} & \cdots & \overline{\hat{f}_{3 S,-S}} \\
\vdots & \ddots & \vdots \\
\overline{\hat{f}_{3-S, S}} & \cdots & \overline{\hat{f}_{3 S, S}}
\end{array}\right]
$$

equivalently,

$$
1 \tilde{F}_{2}=D^{\dagger} \tilde{F}_{3}^{\dagger},
$$

where 1 is the square matrix with all its elements equal to one, $\tilde{F}_{3}^{\dagger}$ is equal to the right hand side of Equation 50 , and $D$ is the diagonal matrix with diagonal elements $D_{n n}=1 / \hat{f}_{10, n}, n=-S, \ldots, S$.

Thus, Equation 24 can be approximated in Fourier domain as follows:

$$
\mathbf{1} \tilde{F}_{2}(p)=D(p)^{\dagger} \tilde{F}_{3}(p)^{\dagger}
$$

Note that, $D^{\dagger}(p) \tilde{F}_{3}(p)$ can be viewed as the modified measurements and $\mathbf{1}$ as the blurring filter in Fourier domain.

\section{NUMERICAL EXPERIMENTS AND DISCUSSION}

Recall that $f_{3}^{*}=f_{2} * f_{1}^{*}(g)$ where $f_{1}$ is the blurring filter, $f_{3}$ is the projections and $f_{2}$ is the unknown image. In our numerical experiments, we implemented the following Wiener filter:

$$
\hat{W}_{\text {opt }}(p)=\tilde{F}_{1}(p)\left[\tilde{F}_{1}^{\dagger}(p) \tilde{F}_{1}(p)+\sigma^{2} I(p)\right]^{-1} .
$$

where $\tilde{F}_{1}=\mathbf{1}$. The absorption coefficients of the object are reconstructed by

$$
\tilde{f}_{2}(p)=\mathcal{F}^{-1}\left\{\hat{W}_{\text {opt }}\left[D^{\dagger} \tilde{F}_{3}^{\dagger}\right]\right\}
$$

where $D^{\dagger} \tilde{F}_{3}^{\dagger}$ is the Fourier transform of modified measurements.

In our numerical experiments, we approximated the blurring filter, $f_{1}$ as a one pixel wide windowing function. This filter can be viewed as the Kronecker delta function extended along the $r_{2}$ direction. Forier transform of $f_{1}$ was numerically computed using the Fourier transform algorithm given in Section 5.2. When $\hat{f}_{10 n}$ is close to zero, $D_{n n}$ values are very high, making $D$ ill conditioned. For small values of $\hat{f}_{10 n}$, i.e., $\hat{f}_{10 n}<\varepsilon, D_{n n}$ values are replaced by one. $D$ can be viewed as a pseudoinverse of $\hat{f}_{1}$.

We used modified Shepp-Logan phantom and performed two sets of experiments. In the first set of experiments, we investigated how different choice of matrix $D$ effects the performance of the reconstructed images. In the second set, we compare our results with the FBP algorithm. In both set of experiments we have used a modified Shepp-Logan phantom of size $N \times N$. $N$ was choosed to be an odd integer and the rotations of discrete motion subgroup was choosed as $\theta_{j}=\frac{2 \pi j}{N}, j=\frac{-(N-1)}{2}, \ldots, \frac{N-1}{2}$. Functions $f\left(r_{1}, r_{2}, \theta\right)$ of $N \times N \times N$ are zero padded to $(2 N+1) \times(2 N+1)$ along $\mathbb{R}^{2}$ for each fixed $\theta$, to prevent aliasing.

Our numerical studies has shown that the interpolation method used in the second step of the $S E(2)$ Fourier transform is crucial in terms of final image quality. We used two interpolation techniques: nearest neighborhood and linear interpolation. All examples presented here are based on linear interpolation due to its superior performance. The computational complexity of the algorithm is $O\left(S^{3} \log \left(S^{3}\right)\right)$, whereas traditional FBP is $O\left(S^{3}\right)$. 
(a) $\sigma=10^{-0}, \varepsilon=10^{-4}$
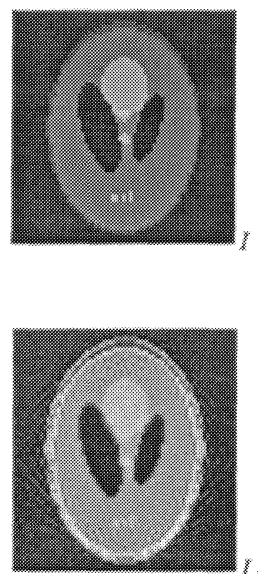

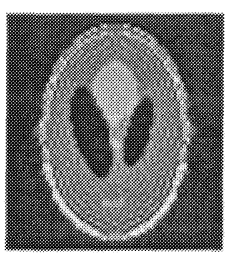

(f) $\sigma=10^{-0}, \varepsilon=10^{-6}$

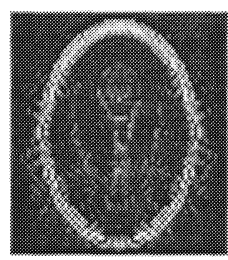

(b) $\sigma=10^{-2}, \varepsilon=10^{-4}$

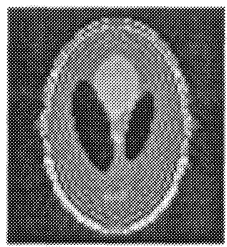

(g) $\sigma=10^{-2}, \varepsilon=10^{-6}$

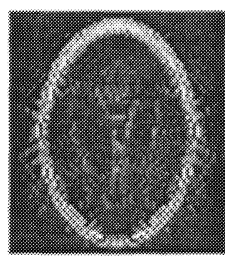

(c) $\sigma=10^{-4}, \varepsilon=10^{-4}$

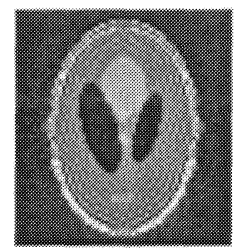

(h) $\sigma=10^{-4}, \varepsilon=10^{-6}$

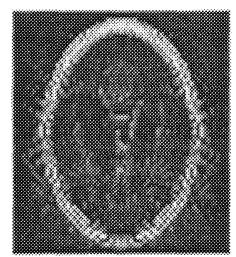

(d) $\sigma=10^{-6}, \varepsilon=10^{-4}$

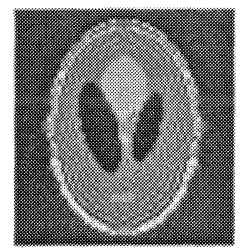

(i) $\sigma=10^{-6}, \varepsilon=10^{-6}$

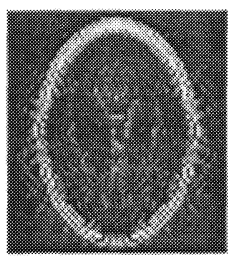

(e) $\sigma=10^{-8}, \varepsilon=10^{-4}$

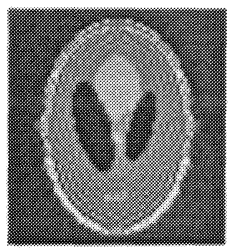

(j) $\sigma=10^{-8}, \varepsilon=10^{-6}$

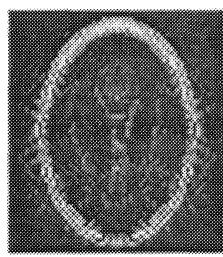

Figure 1. $(I)$ Modified Shepp-Logan phantom of size $65 \times 65$ reconstructed with $(I I)$ FBP and $(a)-(j)$ results of Wiener filtering for $\sigma=10^{-2}, 10^{-4}, 10^{-6}, 10^{-8}$ with thresh hold $\varepsilon=10^{-4}, 10^{-6}$.

For the case $N=65$, the filter was implemented as in Equation 53 for $\sigma$ values equal to $10^{-2}, 10^{-4}, 10^{-6}, 10^{-8}$ and threshold value of $\varepsilon$ equal to $10^{-4}, 10^{-6}$. The results are shown in Figure 1.

We compute the root mean square (RMSE) and peak signal to noise ratio (PSNR)

$$
\begin{gathered}
R M S E=\sqrt{\frac{1}{N^{2}} \sum_{i, j=1}^{N}\left[f_{i j}-\tilde{f}_{i j}\right]^{2},} \\
P S N R=20 \log _{10}\left(\frac{1}{R M S E}\right),
\end{gathered}
$$

of reconstructed images. The results are presented in Table tab: RMSE and PSNR phantom 65 comparison.

\begin{tabular}{|c|c|cc|c|c|c|c|}
\hline & FBP & Proposed method for $\sigma^{2}=$ & 1 & $10^{-2}$ & $10^{-4}$ & $10^{-6}$ & $10^{-8}$ \\
\hline RMSE & 16.3348 & $\varepsilon=10^{-4}$ & 13.7452 & 13.7452 & 13.7452 & 13.7452 & 13.7452 \\
\hline & & $\varepsilon=10^{-6}$ & 23.1200 & 23.1200 & 23.1200 & 23.1200 & 23.1195 \\
\hline PSNR & -24.2623 & $\varepsilon=10^{-4}$ & -22.7630 & -22.7630 & -22.7630 & -22.7630 & -22.7630 \\
\hline & & $\varepsilon=10^{-6}$ & -27.2797 & -27.2797 & -27.2797 & -27.2797 & -27.2796 \\
\hline
\end{tabular}

Table 2. RMSE and PSNR comparison for phatom $65 \times 65$

Numerical studies show that RMSE and PSNR do not depend on the choice of $\sigma$. When compared with FBP algorithm, with the treshold value $\varepsilon=10^{-4}$ proposed algorithm has lower RMSE for $\sigma^{2}=10^{-4}$. We applied the filter on a phantom of size $129 \times 129$ with these parameters. Figure 2 shows that the reconstructed images for the proposed filter (Fig. 2(c)) and the FBP (Fig. 2(b)). Visual comparison shows that the proposed algorithm produces results that are as good or better than FBP algorithm. Note that the contrast of the second image appears to be better than that of FBP (Fig.2). All boundaries are sharper and more consistent as compared with the FBP. Additionally RMSE and PSNR values of the proposed algorithm is better than that of FBP (Table 3).

\section{CONCLUSION}

We developed an algorithm based on a group theoretical Wiener filtering framework to invert the Radon transform. Proposed algorithm produced comparable or better results relative to FBP algorithm. In the future, we will report the robustness of this algorithm in the presence of noise, and applications in radiation therapy treatment planning. 

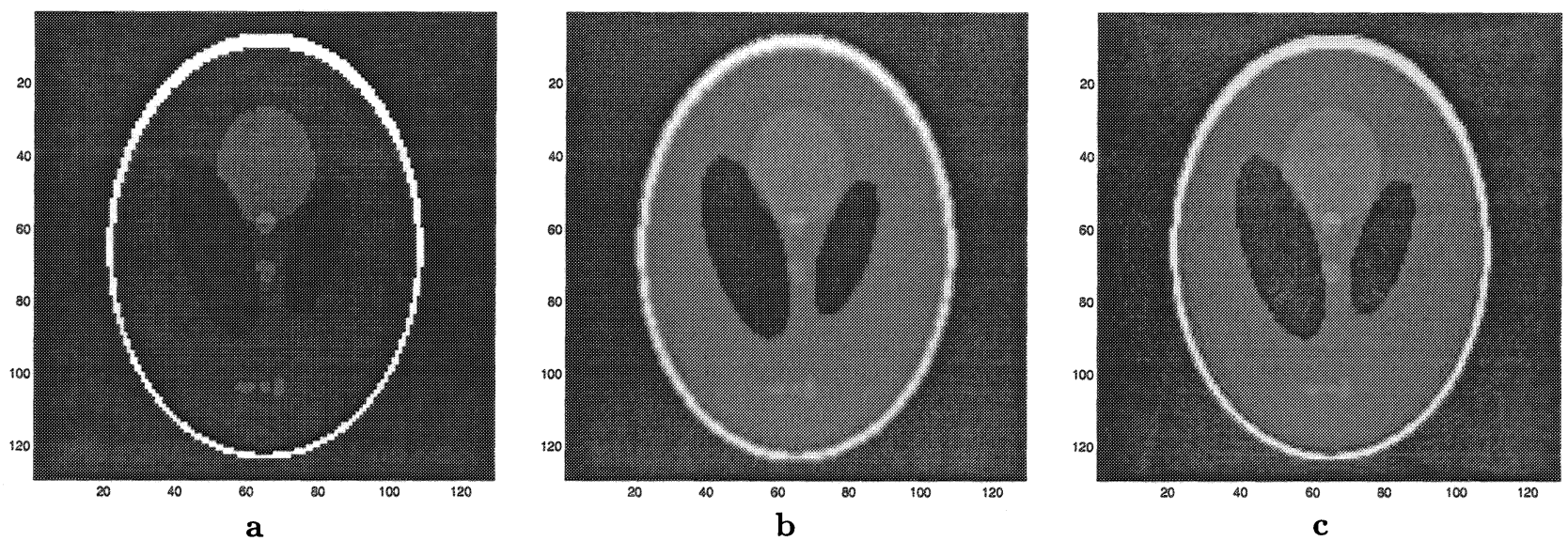

Figure 2. (a) Modified Shepp-Logan phantom of size $129 \times 129$ is (b)reconstructed using FBP (c) and proposed algorithm for $\sigma^{2}=10^{-} 4, \varepsilon=10^{-} 2$

\begin{tabular}{|c|c|c|}
\hline & FBP & Proposed \\
\hline RMSE & 7.8259 & 7.7234 \\
\hline PSNR & -17.8707 & -17.7562 \\
\hline
\end{tabular}

Table 3. RMSE and PSNR comparison for phantom $129 \times 129$

\section{REFERENCES}

1. C. Barber, D.P. Dobkin, H. Huhdanpaa, "The quickhull algorithm for convex hulls," ACM Trans. Math. Soft., 22(4), pp. 469-483, 1996.

2. S. Basu, Y. Bresler, " $O\left(N^{2} \log _{2} N\right)$ Filtered back projection reconstruction algorithm for tomography", IEEE Transactions on Image Processing, 9(10), 1760-1773, 2001.

3. L. Brady, "A fast Discrete Approximation Algorithm for the Radon transform," SIAM J. of Comput., 27(1), 107-119, 1998.

4. G.S. Chirikjian, A.B. Kyatkin, Engineering Applications of Noncommutative Harmonic Analysis, CRC Press, 2000.

5. F. Cools, G.C. Hermann, R.M. Van Der Weiden, F.B. Kets, "Fast Computation of the 3-D Radon transform," Geophysics, 62(1), pp. 362-364, 1997.

6. S.R. Deans, The Radon transform and some of its applications. Wiley Interscience, 1983.

7. M. Defrise, R. Clack, D.W. Townsend, "Image reconstruction from truncated, two dimensional, parallel projections," Inverse Problems, 11, pp. 287-313, 1995.

8. A. Grosmann, J. Morlet, T. Paul, "Transforms associated to square integrable group representations. I. General results," J. Math. Phys., 26(10), pp. 2473-2479, 1985.

9. .E.J.Hannan, Group representations and applied probability. Methuen \& Co., Ltd, London, 1965

10. S. Helgason, The Radon Transform, Progress in Mathematics, Vol. 5, Birkhauser, 1980.

11. P. Kuchment, K. Lancaster, L. Mogilevskaya, "On local tomography," Inverse Problems, 11, pp. 571-589, 1995.

12. A.B. Kyatkin, G.S. Chirikjian "Patern matching as a correlation on the discrete motion group," Comp. Vis. Img. Und., 74(1), pp. 22-35, 1999.

13. M. Sugiura, Unitary representations and harmonic analysis -an introduction-, Halsted Press, 1975.

14. N.J. Vilenkin, Special functions and the theory of representations. American Mathematical Society, 1988.

15. A.M.Yaglom, "Second ordeer homohenous random fields", Fourth Berkeley Symposium on Mathematical Statistics and Probability, University of Califonia Press, Berkeley, pp.593-622, 1961

16. B. Yazıc1, "Stochastic Deconvolution over Groups - Part I", submitted to IEEE Transactions in Information Theory, April 2002. 